$$
\text { CONF-950956--3 GA-A22189 }
$$

\title{
DIAGNOSTICS FOR \\ THE DIII-D RADIATIVE DIVERTOR
}

by

D.G. NILSON, N.H. BROOKS, J.P. SMITH, and R.T. SNIDER 


\section{DISCLAIMER}

This report was prepared as an account of work sponsored by an agency of the United States Government. Neither the United States Government nor any agency thereof, nor any of their employees, makes any warranty, express or implied, or assumes any legal liability or responsibility for the accuracy, completeness, or usefulness of any information, apparatus, product, or process disclosed, or represents that its use would not infringe privately owned rights. Reference herein to any specific commercial product, process, or service by trade name, trademark, manufacturer, or otherwise, does not necessarily constitute or imply its endorsement, recommendation, or favoring by the United States Government or any agency thereof. The views and opinions of authors expressed herein do not necessarily state or reflect those of the United States Government or any agency thereof. 


\section{DISCLAMMER}

Portions of this document may be illegible in electronic image products. Images are produced from the best available original document. 


\title{
DIAGNOSTICS FOR THE DIII-D RADIATIVE DIVERTOR
}

\author{
by \\ D.G. NILSON, ${ }^{*}$ N.H. BROOKS, J.P. SMITH, \\ and R.T. SNIDER
}

This is a preprint of a paper presented at the 16th IEEE/NPSS Symposium on Fusion Engineering, September 30-October 5, 1995, Champaign, Illinois, and to be printed in the Proceedings.

\author{
Work supported by \\ U.S. Department of Energy Contracts \\ W-7405-ENG-48 and DE-AC03-89ER51114
}

*Lawrence Livermore National Laboratory

GENERAL ATOMICS PROJECT 3466

OCTOBER 1995 


\title{
Diagnostics for the DIII-D Radiative Divertor*
}

\author{
D.G. Nilson, ${ }^{\text {a }}$ N.H. Brooks,, J.P. Smith,, R.T. Sniderb \\ aLawrence Livermore National Laboratory, P.O. Box 808, Livermore, California 94551-9900

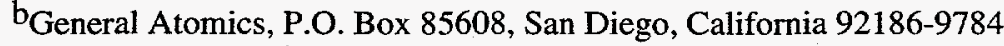

\begin{abstract}
This paper reviews the design of new diagnostics and the modifications to existing diagnostics needed to carry out radiative divertor experiments in DIII-D following installation in late 1996 of a set of baffle structures that will restrict the backflow to the core plasma of neutral deuterium atoms and impurity gases. The divertor slots formed by the new baffle structures will inhibit the easy view of the divertor legs and target plates that the open divertor geometry in DIII-D currently affords. We review a basic set of diagnostics that are needed to demonstrate the reduction of divertor heat loading and radiative dissipation of energy within the divertor. This will include IR cameras, bolometry, foil bolometers, and Langmiur probes. Within the limits of available funding, we will implement a supplemental set of instruments which provide a more detailed understanding of the underlying physical processes. Many existing diagnostics require only re-aiming to provide proper coverage of the initial $23 \mathrm{~cm}$ long divertor plasma configuration (X-point to floor distance). Other diagnostics need extensive reconfiguration using in-vessel fiber-optic bundles or high power laser mirrors. The new divertor baffle panels provide a protective shelf for diagnostic hardware mounted underneath them, but the water cooling channels in the panels limit the permissible size of through holes and, thereby, restrict the available views of under-the-baffle diagnostics. The successful resolution of the design and implementation of these diagnostic modifications is dependent on a strong coordination between GA and its many diagnostic collaborators.
\end{abstract}

\section{INTRODUCTION}

The primary purpose of the radiative divertor program (RDP) is to modify the existing DIII-D divertor structure in order to allow integrated testing of divertor heat-flux reduction concepts and advanced tokamak operating scenarios. Specifically, the top/bottom symmetric divertor structure is designed to match the shape of proposed high-triangularity double-null plasmas with high confinement and stability in order to provide pumping for density control of these plasmas and to baffle the leakage of gas from the divertor plenum into the main plasma. The hardware will consist of extended baffles near the outer divertor strike points (top and bottom) and a raised central baffle in the private flux region under each $\mathrm{X}$-point. Each baffle region will have a separate liquidhelium-cooled cryopump. A cross section appears in Fig. 1. In the future, the depth of the divertor slot can be increased from $23 \mathrm{~cm}$ to $43 \mathrm{~cm}$, as measured along the separatrix from $\mathrm{X}$-point to target plate.

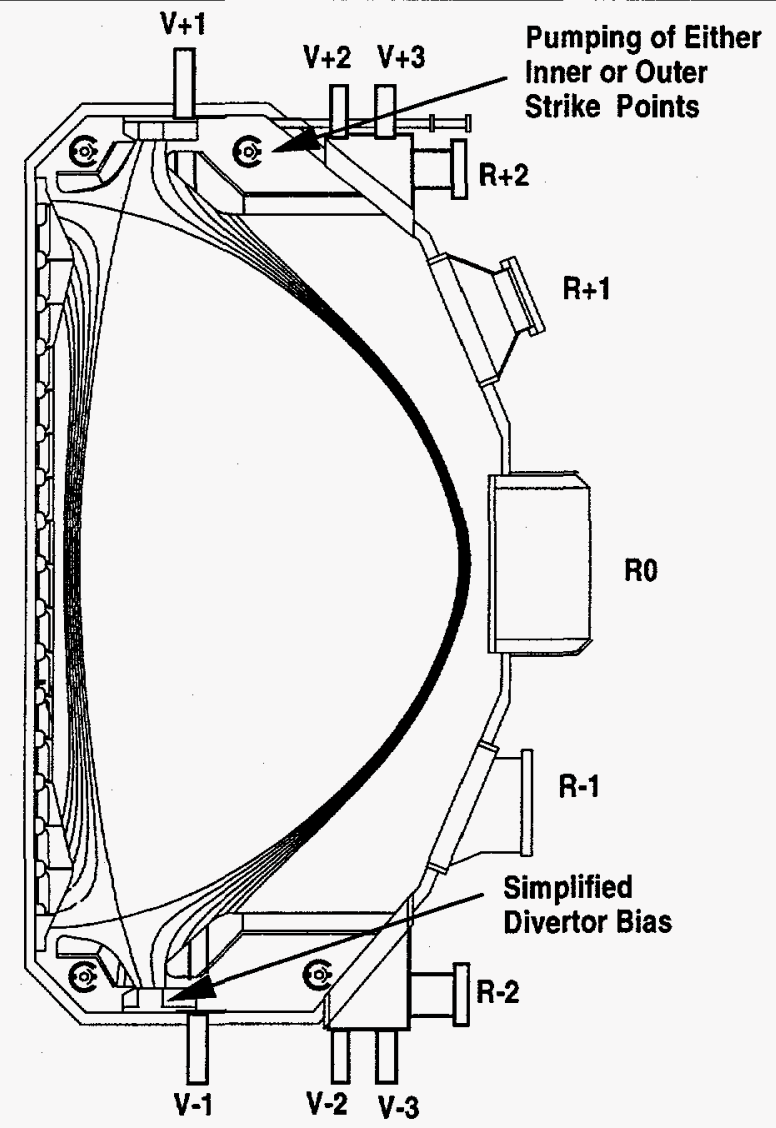

Fig. 1. Planned radiative divertor upgrade for DIII-D.

The radiative divertor will impact divertor diagnostics in several ways. First, the increased emphasis on producing highly radiative plasmas using impurity injection pushes spectroscopy to the forefront and demands more detailed multi-point or multi-chord measurements in the divertor region. Second, understanding impurity transport requires some knowledge of the background plașma flow in order to estimate the frictional force on impurity ions and new diagnostics which measure plasma flows must be installed on DIII-D. Third, the addition of new baffle plates and cryopumps means that many diagnostics will have to be relocated and that others will need new views just to maintain existing capability. This impact is minimized for the first configuration with the $23 \mathrm{~cm}$ deep slot, but with the deep $43 \mathrm{~cm}$ slot virtually all divertor measurements will be impacted to some degree. Finally, by choosing to run primarily double-null divertors, diagnosis of the upper divertor must be expanded to provide key information

*Work supported by the U.S. Department of Energy under Contract No. DE-AC03-89ER51114 and W-7405-ENG-48. 
comparable to that presently obtained only in the lower divertor.

The basic set of diagnostics proposed for the RDP is nearly the same as those now in place on DIII-D (See Table I). These diagnostics will provide measurement of the first wall heat flux using infrared TVs, the radiative power loss via two 24 channel bolometer arrays [2] plus two new arrays looking at just the lower divertor, and the particle flux to the divertor targets using arrays of fixed Langmuir probes [3]. Neutral pressure in the pumping plenums will be measured with fast responding (5 ms) ASDEX type neutral pressure gauges [4]. In addition, visible TVs and spectrometers [5,6] will monitor line emission from the plasma and plasma facing components in the divertor region. Central impurity densities can be inferred from VUV spectroscopy [7] and CER [8] measurements near the plasma midplane. If possible, the divertor Thomson system [9] will be modified to allow continued measurements of $n_{e}$ and $T_{e}$ between the $X$-point and one divertor target. All of the diagnostics in Table I are currently in use or will be installed by June 1996 on the existing divertor configuration. Though many of them can be adapted for the radiative divertor with no change in their basic design, their in-vessel components will have to be rebuilt with entirely new hardware. Examples of diagnostics in this category are the " $2 \pi$ " foil bolometers [10], fixed Langmuir probes, tile current arrays [11], divertor interferometers [12] and reflectometers [13]. Others will have to be modified significantly to maintain a suitable viewing geometry.

Much of the diagnostic modifications will be carried out by the following collaborating institutions as they have unique physics and engineering resources:

\section{Lawrence Livermore National Laboratory \\ Oak Ridge National Laboratory \\ Sandia National Laboratory \\ Princeton Plasma Physics Laboratory \\ University of California at San Diego \\ University of California at Los Angeles \\ Johns Hopkins University}

General Atomics has a strong history of successful collaborations with these institutions on DIII-D and will rely on them again to field the complex diagnostics required for the RDP.

\section{HEAT FLUX MEASUREMENTS AND RADIATIVE LOSSES}

An array of six calibrated infrared cameras (IRTV's) measure the surface temperature, which we use to infer the local heat flux. The cameras record 8-12 $\mu \mathrm{m}$ light emission with $125 \mu \mathrm{s}$ temporal resolution and $0.5 \mathrm{~cm}$ spatial resolution. For the $23 \mathrm{~cm}$ divertor slot, we will make relatively minor changes to the existing IRTV systems. Only some re-aiming of routing mirrors will be required.

We will upgrade the data acquisition for two cameras. Currently, we record the signal from each camera on a VCR during the shot and replay the tapes one at a time through a video digitizer after the shot. In place of the two-step process, we will substitute a frame-grabber, digitize the live video signal during the shot, storing the relevant part of the image. This will eliminate the non-linearity and signal degradation introduced by tape recording. The video can still be taped for archival storage. Direct digital acquisition will speed access to the data after the shot. From the measurements of brightness, the temperature change of the tile surfaces is extracted and converted to heat flux. The total power to the first wall is estimated by assuming toroidal symmetry. Peak power versus time and total power versus time can be available a few minutes after the shot, allowing session leaders to determine gas puff quantities for subsequent radiative divertor experiments.

Plasma radiative losses are measured with a set of two bolometer arrays of 24 viewing chords each. The arrays are currently located at an $\mathrm{R}+2$ and $\mathrm{R}-1$ port, with each providing complete poloidal coverage of both the core and divertor plasma. With the arrays at different poloidal locations, unfolding of the $2 \mathrm{D}$ radiation profile has been possible. For the new RDP configuration, the bolometer array currently located at an $\mathrm{R}+2$ port will be moved to an $\mathrm{R}+1$ port as shown on the left-hand side of Fig. 2. This will make the bolometer arrays up/down symmetric. From its respective location each array should be able to view the opposing divertor.

Higher spatial resolution will be required in the divertor region and will be accomplished by adding two arrays of 6-7 detectors each under the lower divertor structures. They will view the divertor plasma through two relatively small

Table I

Key Diagnostics for the Radiative Divertor Program

(*denotes modification needed, **denotes new diagnostic)

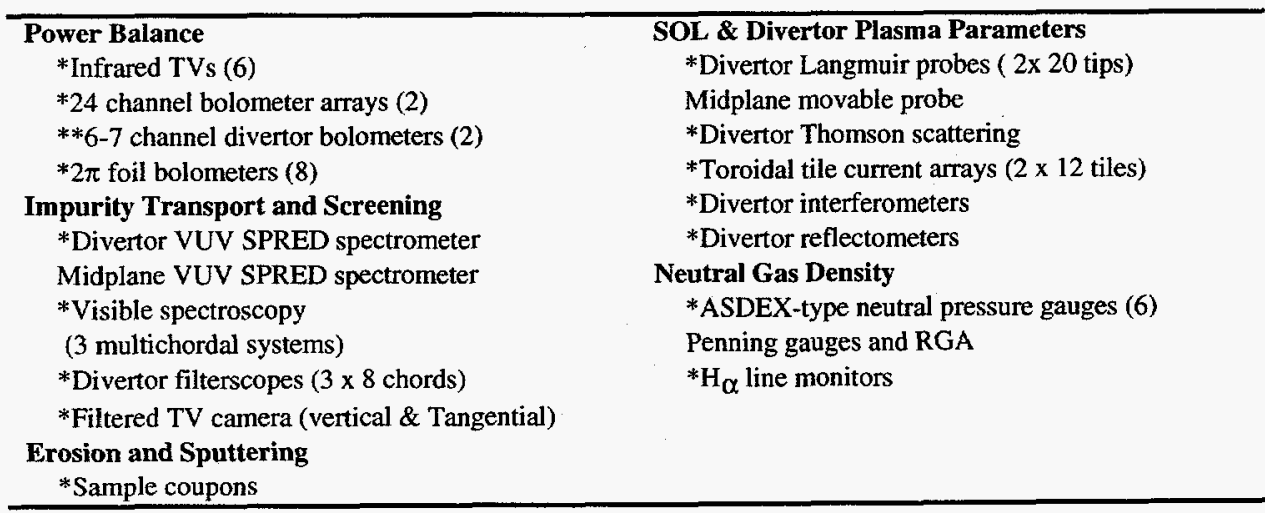




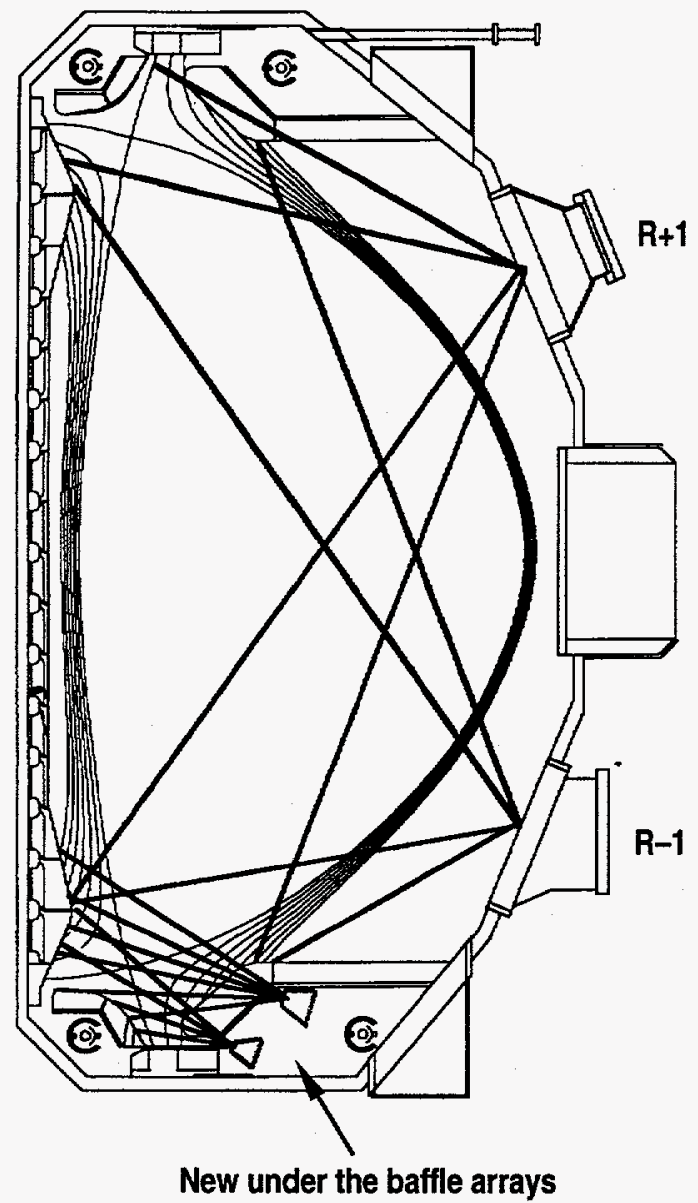

Fig. 2. Additional bolometer arrays under the divertor baffles improve the spatial resolution attainable with tomographic inversion.

openings in the structure as outlined on the right in Fig. 2. This should give a spatial resolution of $\sim 2 \mathrm{~cm}$ in the outboard leg of the divertor. The new detectors will be of the same type as in the existing arrays, each consisting of a $0.25 \mathrm{~cm}^{2}$ platinum absorbing layer laminated to a $50 \mathrm{~W}$ platinum meander-path resistor insulated with Kapton. The temperature of the sensor increases with incident radiated power and particle flux and is detected as an increase in resistance of the sensor. The sensors can withstand temperatures of about $200^{\circ} \mathrm{C}$ and will require active cooling during high temperature vessel baking. Important issues in the design of this divertor radiation diagnostic include positioning of the sensors and design of openings in the divertor structure to produce the best viewing angles to unfold the radiation distribution. To house the sensors, an integral support structure will be designed that provides a viewing aperture and a stable viewing position through the divertor openings.

Additional information on the location and nature of the radiated power is provided by an array of foil bolometers. Thin graphite foils ( $\sim 0.05$-in. thick) are attached to a plasmafacing graphite tile surface. Radiated power impinging on the foil surface is deduced from the time-dependent behavior of its surface temperature [1] Although recessed slightly inside a tile for protection, each foil has virtually the same exposure as the tile itself, and for that reason the foil has also been referred to as a " $2 \pi$ bolometer." Foil placement is considered at each poloidal location where divertor IR camera systems are already planned ( 2 toroidal locations for the lower divertor and one location for the upper divertor).

\section{DIVERTOR AND SCRAPE-OFF-LAYER (SOL) CONDITIONS}

\section{A. Fixed Probes}

The use of fixed Langmuir probes, in conjunction with infrared measurements, will be used to determine directly the extent of the heat and particle flux reduction. In addition, the electron temperature and plasma density measurements made with the probes will be used to understand and model the detachment process. These techniques also provide information on the structure of the divertor plasma sheath which is now recognized to be strongly modified by the local neutral pressure obtained in gaseous divertor experiments. We will need to make more detailed and localized measurements than with the open divertor due to the geometrical constraint placed on the plasma by the RDP baffle hardware. Each array would consist of 5-6 probes at the inner strike point, $2-3$ in the private flux region, 5-10 at the outer strike point, and 5 on the outer baffle. Toroidally offset probes will be needed to achieve adequate spatial resolution of the profiles. We plan to install (but not fully instrument) a mirror set of probes in the upper divertor. Both the upper and lower probe arrays would have an identical number of probes at one toroidal location. There would be 5 additional probes spread toroidally ( $n=6$ ) near the lower divertor outer strike point (OSP) to measure toroidal asymmetries

\section{B. Tile Current Monitors}

Tile current monitors have been used to measure the magnitude, sign and toroidal distribution of SOL currents, and in quantifying halo currents during disruptions. In the RDP configuration, the forces from toroidally asymmetric disruption halo currents generate the most severe forces to which the baffle structure is subjected. Emphasis has therefore been shifted from monitoring SOL currents in normal plasma operation to measuring the magnitude and toroidal distribution of halo currents during disruptions. Fortunately, the halo currents in the RDP configuration are intercepted by the tiled surface of the outer baffle plates and channeled through the support legs of this structure to the vessel wall before closing their poloidal circuit. The channeling of the current through the support legs affords a much easier method of measurement than was possible in the ADP configuration where these currents passed mainly through tiles mounted directly to the vessel surface. Rogowski coils will be placed around the support legs of the outer baffle to measure the halo current and to impose operational limits on the plasma current driven in the main plasma.

\section{Thomson Scattering}

The new divertor Thomson diagnostic provides crucial plasma temperature and density information. Its collection optics are installed on an R-1 port and view one of the eight 
laser beams of the core Thomson system that has been rerouted into a neighboring vertical port. Unfortunately, the optimum plasma shape and resulting tile surfaces to study radiative divertor physics requires that a shelf cover the port used for the divertor Thomson laser beam. This shelf would also obstruct the view to the divertor of the current collection optics. A clever method must be devised to re-direct the beam to probe the region of the outer lower divertor leg. Possible solutions might require internal mirrors on either the top or bottom of the vessel as well as internal beam dumps. Sufficient light collecting efficiency, low stray light levels and alignment verification must be maintained. Cleanliness of optical components mounted inside the vessel is also a major concern due to the possible stray light and high laser energy densities on the mirrors. Initial layouts indicate that the existing collection optics would probably maintain sufficient light collecting efficiency provided the proper outer shelf tile modifications are allowable.

\section{Interferometry And Reflectometry}

To provide density information in the new radiative divertor, it is planned to include both interferometer and reflectometer systems. These systems will be complementary, and are designed to provide line integrated density, density profiles, peak density, and density turbulence measurements in the divertor. In each case it is intended to upgrade or improve the systems in the light of actual operational experience in the new divertor configuration on DIII-D.

Possible configurations for the interferometer and reflectometer in the radiative divertor are shown schematically in Fig. 3. The added baffle structures in the RDP provide more flexibility in locating microwave horns and components than presently exists, and provides better access and views of the divertor legs and strike points. Since positioning of the divertor legs is more constrained, it is easier to design the systems such that the probe beams are perpendicular to the density gradients.

\section{IMPURITY CONCENTRATION AND LINE BRIGHTNESS}

\section{A. VUV Spectroscopy}

A SPRED XUV Spectrometer has recently been installed to measure the impurity ion density in the divertor. The impurity ion density can be determined from the measured impurity brightness, using electron impact excitation cross sections and measured electron density and temperature. For XUV excited states, the electron impact excitation cross sections are well known and the electron temperature and density will be measured using the divertor Thomson diagnostic. The SPRED spectrograph is installed on a top vertical port, so that the column-integrated brightness of impurity ions are measured along a vertical viewchord with the same major radius as that of the divertor Thomson laser beam. To obtain radially-resolved spatial profiles of impurity ion densities, the plasma position is swept across the spectrograph field of view. We use the LLNL SPRED spectrograph to observe the $10-30 \mathrm{~nm}$ and $10-100 \mathrm{~nm}$ regions, respectively with $0.04 \mathrm{~nm}$ and $0.2 \mathrm{~nm}$ spectral resolution in the
DIII-D divertor. A multi-channel detector system under microcomputer control allows measurement of time-resolved spectra. To continue to use this diagnostic during the RDP experiments will require that both the SPRED instrument and its associated port tube be tilted to view the outer leg. Initial studies suggest that a tilted line of sight can be achieved without sacrificing an excessive fraction of the spectrometer's light collection cone.

\section{B. Soft X-Ray Spectroscopy}

Multi-Layer Mirror (MLM) technology provides a means of obtaining very high reflectivity in the soft $\mathrm{X}$-ray spectral range of 1-10 nm and performing spectroscopy of the ionic resonance lines of low- $Z$ elements. Measuring the absolute intensities of these resonance lines is essential to diagnosing impurity abundance in the divertor.

John Hopkins University (JHU) has developed a compact, invacuum MLM spectrometer [14] for use on the C-Mod tokamak. A fast phosphor serves as a signal transducer, converting the soft $\mathrm{X}$-ray radiation into visible light which is conveyed by fiberoptics across a vacuum window for remote detection outside the tokamak enclosure. Installation of similar devices on DIII-D requires some change in materials to meet DIII-D's stringent vacuum regulations and its $400^{\circ} \mathrm{C}$ bakeout temperature. JHU proposes to install two or three such compact MLM devices in an R-1 port on DIII-D with radial views across the divertor region, both above and below the X-point. During a clean vent, the flange-mounted diagnostic could be modified to view lines of neon or argon, instead of carbon. Installation in an R-1 port has been chosen as an initial step toward certifying this diagnostic for future use under the baffle with views similar to that of the bolometers depicted in Fig. 2.

\section{Divertor Visible Spectroscopy}

The term Divertor Visible Spectroscopy encompasses the existing $D_{\alpha}$ monitors/filterscopes, survey spectrometers, and high resolution spectrometers. The filterscopes are an upgrade to the existing $\mathrm{D}_{\alpha}$ monitors that will permit monitoring three different spectral lines simultaneously from the light delivered by a single fiber. Prior to the RDP upgrade, DIII-D will have two arrays of such spectral monitors installed: a near-vertical fan of viewchords viewing the upper divertor and a similar fan viewing the lower divertor. DIII-D will have three visible spectrometers viewing the divertor prior to the RDP. These instruments are the Multichordal Divertor Spectrometer, the Russian high resolution spectrometer and the Divertor Visible SPRED. Collectively, these instruments enable the localization of impurity radiation and the measurement of doppler profiles.

All these systems share the common feature that their detector systems are located outside the DIII-D radiation enclosure and rely on quartz fiberguides to convey light from tokamak to diagnostic. At present, plasma light is input to the quartz fiberguides with collection lenses mounted external to vacuum windows. In most cases it will be possible to preserve this simple design simply by re-aiming the collection lens/fiber assembly or by manufacturing a new 


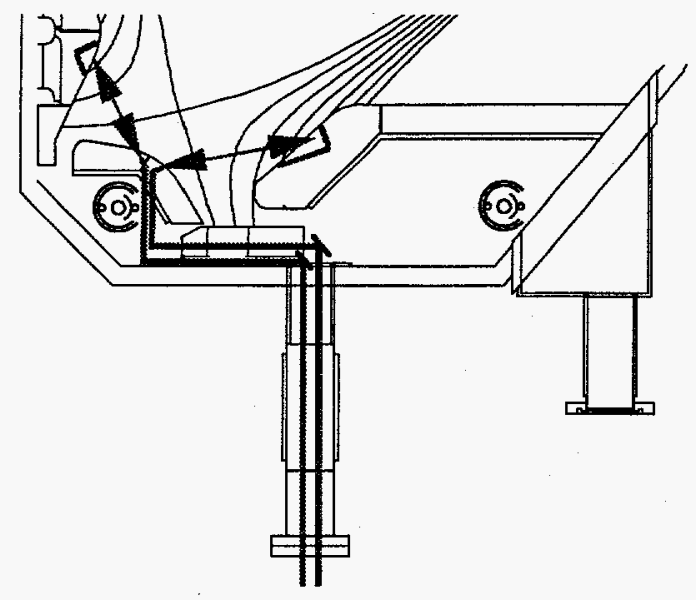

Divertor Interferometer

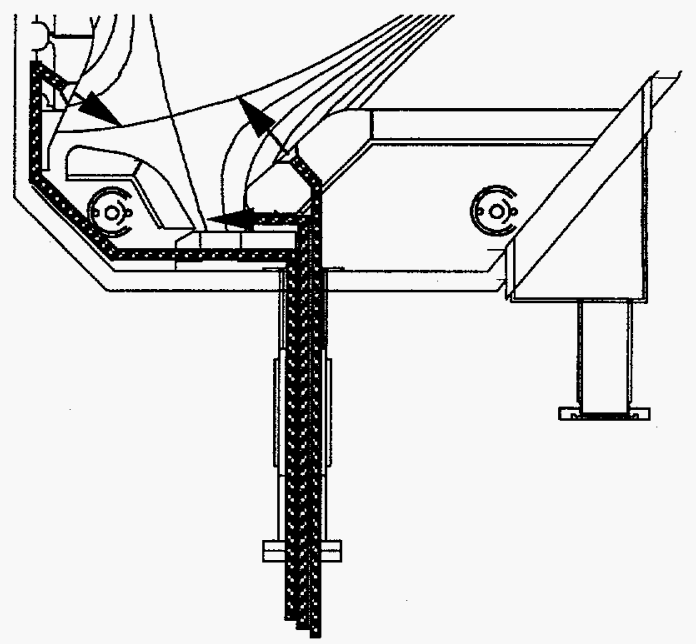

Divertor Reflectometer

Fig. 3. Interferometer and reflectometer paths crossing the divertor legs give line average and peak electron density with high time resolution.

fiber clamp that repositions the fibers behind the lens. Unfolding the spatial location of radiation in the divertor region will require new, near-horizontal viewchords that cross the existing fans of near-vertical viewchords. The use of in-vessel fiberoptic telescopes is attractive for this purpose. An array of six telescopes will be installed under the outer baffle in both the lower and upper divertor plenums. Each array will have a poloidal fan of views as shown in the Fig. 4. The graphite tiles on the slanted baffle will be slotted poloidally to pass light from the divertor region to the telescopes mounted behind the pump entrance of the outer divertor plenum. Identical mounting structures for the telescopes will be built for the top and bottom divertor, with the exception that a mirror will be required in the upper divertor to avoid interference with the local cryopump. The fiberoptic

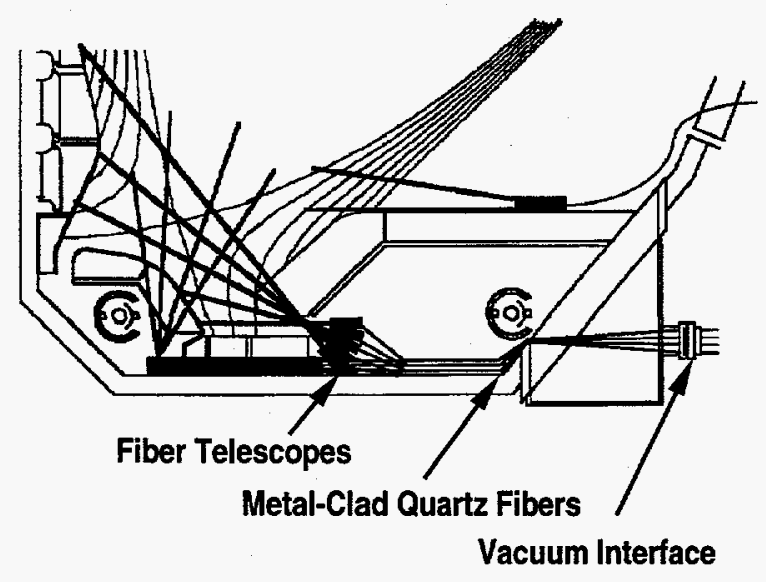

Fig. 4. Use of bakeable fiber telescopes under the baffle enable measurements of visible light close to the outer divertor target plate. vacuum/air coupler will consist of $3 \mathrm{~mm}$ diameter sapphire lenses brazed into a vacuum flange. The in-vacuum hardware employs special metal-clad, quartz fibers that can withstand the $400^{\circ} \mathrm{C}$ bakeout temperature of the vessel. Each fiber will have its own input telescope with long collimator tube to prevent contamination of its collection lens. In the Control annex, patch panels of 3-way fiber splitters will be used to distribute the light from a single fiber telescope to photomultiplier detector channels containing filters for different spectral lines. Though this in-vessel fiberoptic system was conceived specifically for a new array of filterscopes, the design will be useful for all fiberoptically coupled, visible diagnostics.

\section{Visible Plamsa Imaging}

Imaging the divertor plasma in the visible provides real time views of features in the divertor regions, e.g., ELMS, detachment, MARFE formation, plasma position, $\mathrm{X}$-point and strikepoint locations, and locked modes, which are important to consider in the control room when preparing for subsequent shots. Secondly, when correlated with other diagnostics, it can provide information on the cause and effect relationship between various events that are separated by large poloidal distances. Finally, when detailed analysis is done on 2D image data which is absolutely calibrated, it can provide very good benchmarking information for $2 \mathrm{D}$ SOL simulation codes used on ITER divertor designs. The design for the RDP, which builds on the techniques used in the current configuration consists of a vertical re-entrant tube extending through a V-1 port as shown in Fig. 5. The reentrant tube houses a fiber optic image guide that views through a vacuum window and onto a $45^{\circ}$ mirror mounted on the tube end. With the tip of the re-entrant tube extending 


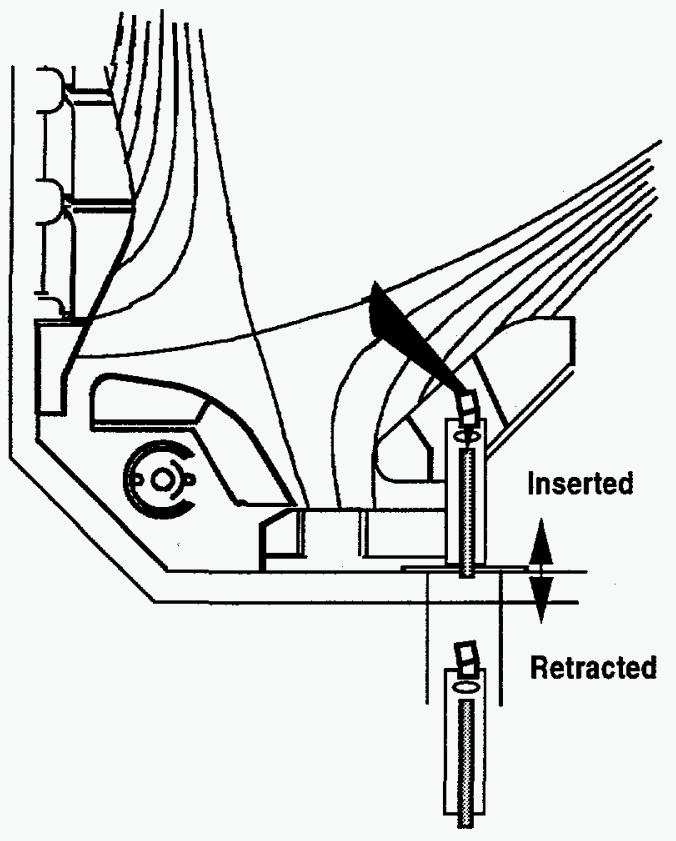

Fig. 5. A retractable re-entrant tube permits continued use of the present imaging optics for tangential TV.

through the slanted portion of the outer baffle and the proper tile removal, this view would give complete coverage of the outer leg. Because the re-entrant tube would remain inside the $\mathrm{V}-1$ port tube when retracted, the image guide would have to be actively cooled in the same way as that currently being implemented for the ADP. To avoid conducting halo currents during disruptions, the re-entrant tube must be electrically insulated from the surrounding tiles and baffle cutout.

Present visible imaging systems on DIII-D use Charge Coupled Device (CCD) or Charge Injection Device (CID) cameras locally in the machine hall to generate the pixel data for the images. This subjects the cameras to a harsh neutron environment, high magnetic fields and field gradients, and strong EMF fields from the dynamics of the tokamak shot. Therefore, cameras are to be placed sufficiently far from the device that the fields are below a threshold value throughout the plasma shot. This will be achieved using flexible, cooled fiber image guides which are currently being used.

\section{NEUTRAL PRESSURE MEASUREMENTS}

Neutral pressure in the RDP will be measured at several locations. Two fast ionization gauge heads (for redundancy) will be placed near each of the four cryopumps (see Fig. 1). These heads and associated electronics are based on the prototype fast ionization gauges developed at ASDEX and will measure the neutral pressure in the pumping plenums. Combined with off-line measurements of the pumping speeds, the data will yield the cryopump exhaust rate for each pump (the product of the pumping speed and neutral pressure), a necessary part of the global neutral particle balance. This information will be used to examine the global particle balance with all four cryopumps, and to compare the effectiveness of inner strike point pumping versus outer strike pumping and lower divertor vs. upper divertor pumping in terms of particle control. Neutral pressure will also be measured in the private flux regions of the upper and lower divertors. This will be accomplished by installation of gauge heads in the gap of the structure above the inner strike point pumps. All of the existing fast ionization gauges installed in DIII-D have been mounted on vessel flanges or re-entrant tubes, whereas the installations for the RDP call for the gauge heads to be mounted directly to the vacuum vessel or RDP baffle structure.

\section{EROSION MEASUREMENTS}

Measurement of erosion and redeposition are obtained with demountable coupons. Small removable samples or coupons will be installed in the divertor tiles for change-out at each invessel vent. With highly polished coupons, direct measurement of the coupon height will yield the cumulative thickness of material eroded or deposited between successive vents. In addition, SIMS and AUGER analysis with argon ion etching will track the detailed history of the deposition, revealing successive boronizations and the presence of any redeposited impurities. The boronization events serve as temporal markers permitting post-facto determination of the layer thickness accummulated in each operating campaign. A poloidal array of coupons spanning the upper divertor, lower divertor and centerpost is planned.

\section{REFERENCE}

[1] D.N. Hill et al., Rev. Sci. Instrum. 59, p. 1878, 1988.

[2] A.W. Leonard, W.H. Meyer, B. Greer, D.M. Behne, and D.N. Hill, Rev. Sci. Instrum. 66, p. 1201, 1995.

[3] D. Buchenauer et al., Rev. Sci. Instrum. 61, p. 2873, 1990.

[4] C.C. Klepper et al., J. Vac. Sci. Technol. A 11, p. 446, 1993.

[5] N.H. Brooks, A. Howald, C.C. Klepper, W.P. West, Rev. Sci. Instrum. 63, p. 5167, 1992.

[6] S. Tugarinov, et al., Rev. Sci. Instrum. 66, p. 603, 1995.

[7] R.J. Fonck, A.T. Ramsey, and R.V. Yelle, Appl. Opt. 21, p. $2115,1982$.

[8] P. Gohil et al., in Proc. of IEEE Symp. on Fusion Engineering, vol. 2, p. $1199,1992$.

[9] T.N. Carlstrom, J.H. Foote, D.G. Nilson, and B.W. Rice, Rev. Sci. Instrum. 66, p. 492, 1995.

[10] T.W. Petrie and J.T. Scoville, Rev. Sci. Instrum. 56, p. 1156, 1985.

[11] T.E. Evans et al., J. Nucl. Mater. 220-222, p. 235, 1995.

[12] R.A. James et al., Rev. Sci. Instrum. 66, p. 422, 1995.

[13] C.W. Domier et al., Rev. Sci. Instrum. 63, p. 466, 1992.

[14] S.P. Regan, M. Finkenthal, M.J. May, and H.W. Moos, Rev. Sci. Instrum. 66, p. 770, 1995. 

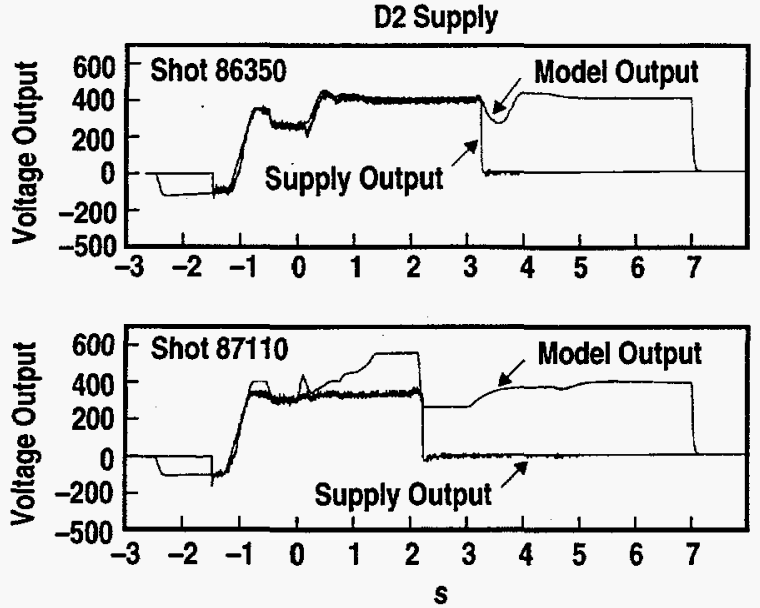

Fig. 4. Example output of model comparison program for F-power supply D2.

\section{E. Power Supply Command, Feedback, and Data Acquisition Signals}

Calibration procedures for the power supply and chopper systems has in the past been a laborious process requiring a high allocation of technician time and test equipment. Systems were checked individually while measurements were made and recorded by hand, increasing the possibility of error. New procedures are being developed to address these issues.

The viability of a PC based system coupled with a high voltage amplifier and digital storage oscilloscopes is being researched. The PC would function as both a control and data recording tool. Several systems could be calibrated simultaneously increasing the test efficiency. The elimination of manual data recording would increase calibration accuracy.

\section{F. Buss Voltage Monitors}

The buss voltage monitors were recently installed and calibrated. Scheduling for follow-up calibration has yet to be determined. The functionality of the PC based system (see Section E) could be applied to the calibration of these voltage probes.

\section{CALIBRATION AND MODEL MAINTENANCE}

Development of a software based calibration update system is underway. A final step included in the calibration of the hardware systems described will be the entry of calibration measurement data into the PCCMS calibration database. This will provide a centralized reference point for tracking equipment status beyond simply updated calibration values. Examples include, last known calibration dates, personnel involved, modifications to equipment, and calibration schedules. With easy access by technicians, data entry after new calibration adjustments will become a routine part of hardware calibrations. After review of adjustments and calibrations, plasma control models will be updated to represent new calibration values. To also ensure hardware upgrades are done in conjunction with model maintenance, a review of system modifications and their impact on plasma control functionality will be included in future design reviews. The models can then be changed and re-calibrated to reflect the modification. This will maintain the plasma control system model accuracy and eliminate the effect of unknown modifications.

In addition to a software database, we are developing a program to compare daily system performance to that predicted by system models. This facilitates a regular check of model accuracy and automated detection of malfunctioning equipment. The comparison program also provides a fast but less accurate check of diagnostic calibration. In Fig. 4 we show two sample plots of the D2 power supply as generated by the comparison program. Actual power supply output voltage data is plotted on top of simulated output data generated by power supply models. Command signals for the power supplies are used as input to the models. In these example plots, the power supplies were turned off by hardware interlocks after approximately 2.3 seconds. Power supply command signals continued to be generated, as indicated by the model output response plots. Acquired command signal data can also be overlaid on the plots for futher verification or analysis.

An example of the programs use as an error detection tool is shown in the second plot (shot 87110 ). The supply is sent a command signal, verified by the model output response or an overlay of the command signal, and fails to respond as predicted by the model output. There also seems to be a delay in the acquired data during the initial turn on. This is being investiated.

\section{CONCLUSIONS}

A Plasma Control Calibration Maintenance System (PCCMS) is under development in parallel with advances in plasma control. Regular equipment calibrations are a necessity for optimum performance in any system. The regular calibration of DIII-D diagnostic hardware will improve accuracy not only in plasma control but also in the collected data used for plasma analysis. In addition, a software database will maintain a centralized documentation system enabling changes to be tracked while providing a link for control model maintenance. The goal of the PCCMS is to maintain PCS performance through preventive rather than corrective methods

\section{REFERENCES}

[1] M.L. Walker, "Toward Integrated Digital Control of the DIII-D Tokamak," in Proc. 15th IEEE/NPSS Symposium on Fusion Engineering, pp. 119-122, 1993.

[2] E.J. Strait, private communication. 